\title{
TITLE:
}

\section{A Survey of the Stable Marriage Problem and Its Variants}

$\operatorname{AUTHOR}(S)$ :

Iwama, Kazuo; Miyazaki, Shuichi

\section{CITATION:}

Iwama, Kazuo ... [et al]. A Survey of the Stable Marriage Problem and Its Variants.

Informatics Education and Research for Knowledge-Circulating Society, 2008. ICKS 2008. International Conference on 2008: 131-136

\section{ISSUE DATE:}

2008

URL:

http://hdl.handle.net/2433/226940

\section{RIGHT:}

(C) 2008 IEEE. Personal use of this material is permitted. Permission from IEEE must be obtained for all other uses, in any current or future media, including reprinting/republishing this material for advertising or promotional purposes, creating new collective works, for resale or redistribution to servers or lists, or reuse of any copyrighted component of this work in other works.; この論文は出版社版でありません。引用の際には出版社版をご確認ご利用ください。;This is not the published version. Please cite only the published version. 


\title{
A Survey of the Stable Marriage Problem and Its Variants
}

\author{
Kazuo Iwama \\ Graduate School of Informatics, Kyoto University, \\ Yoshida-honmachi, Sakyo-ku, Kyoto 606-8501, Japan \\ iwama@kuis.kyoto-u.ac.jp \\ Shuichi Miyazaki \\ Academic Center for Computing and Media Studies, Kyoto University, \\ Yoshida-honmachi, Sakyo-ku, Kyoto 606-8501, Japan \\ shuichi@media.kyoto-u.ac.jp
}

\begin{abstract}
The stable marriage problem is to find a matching between men and women, considering preference lists in which each person expresses his/her preference over the members of the opposite gender. The output matching must be stable, which intuitively means that there is no manwoman pair both of which have incentive to elope. This problem was introduced in 1962 in the seminal paper of Gale and Shapley, and has attracted researchers in several areas, including mathematics, economics, game theory, computer science, etc. This paper introduces old and recent results on the stable marriage problem and some other related problems.
\end{abstract}

\section{Introduction}

Consider a bipartite graph $G=(U, V, E)$, where $U$ and $V$ are sets of vertices and $E$ is a set of edges. A matching $M$ in $G$ is a subset of $E$ such that each vertex appears at most once in $M$. Bipartite matchings are sometimes interpreted as marriage between men and women: $U$ and $V$ represent the set of men and women, respectively, and an existence of an edge between $m \in U$ and $w \in V$ implies that $m$ and $w$ are acceptable to each other.

In the stable marriage problem (SM for short), each person expresses not only the acceptability but also a preference order of the members of the opposite sex, and an output matching must satisfy the stability condition, which intuitively means that there is no man-woman pair both of which have incentive to elope. (Formal definitions will be given in Sec. 2.)

This problem was first introduced by Gale and Shapley in 1962 in their seminal paper [13], and has received much attention from a lot of researchers in many areas because of its inherent mathematical structure and rich applications to the real world. In fact, the introduction of this problem by Gale and Shapley was motivated by the matching between medical students and hospitals in the US, currently known as NRMP (National Resident Matching Program) [52]. (Readers who are interested in its history can refer to [16].) Within the knowledge of authors, there are three other students-hospitals matching systems that use SM (more precisely, the hospitals/residents problem, introduced in Sec. 6.2); CaRMS [9] in Canada, SPA [58] in Scotland, and JRMP [40] in Japan.

In this paper, we survey classical and recent results on (mainly, but not limited to) the stable marriage problem.

\section{Definitions and Basic Results}

In this section, we formally define the stable marriage problem (SM), and introduce some fundamental results. We consider several variants in this paper, but here we give only the definition of the most basic model, and will give definitions of other variants at each introduction point.

An instance $I$ of SM consists of the same number, say $n$, of men and women. Each person has a preference list that strictly orders all members of the opposite sex. If a man $m$ prefers $w_{1}$ to $w_{2}$, we write $w_{1} \succ_{m} w_{2}$. A similar notation is used for women's preferences.

A matching $M$ of $I$ is a set of disjoint man-woman pairs of $I$. In this section, we consider only perfect matchings, namely, matchings of size $n$. If, in a matching $M$, a man $m$ and a woman $w$ are matched together, we write $M(m)=w$ and $M(w)=m$. We say that a man $m$ and a woman $w$ form a blocking pair for $M$, (or simply, $(m, w)$ blocks $M$ ) if the following three conditions are met: (i) $M(m) \neq w$; (ii) $w \succ_{m} M(m)$; and (iii) $m \succ_{w} M(w)$. A matching 
$M$ is unstable if there is a blocking pair for $M$, and stable otherwise.

Gale and Shapley [13] proposed so-called the GaleShapley Algorithm, which runs in time $O\left(n^{2}\right)$, and always finds a stable matching. Hence, this is a constructive proof of the fact that any instance admits at least one stable matching.

\section{Some Extensions of Preference Lists}

In this section, we consider two natural extensions of preference lists. Recall that in the original SM, each person's preference list must include all members of the opposite sex in a strict order. Apparently, this is inconvenient in applications, especially in a large-scale matching system mentioned previously. Hence, we can naturally consider two natural relaxations, namely, incomplete lists and ties in lists. In the following subsections, we see some properties of the variants allowing each or both of these relaxations.

\subsection{Incomplete Preference Lists}

In this variant, each person's preference list may be incomplete, i.e, a person can exclude some members whom he/she does not want to be matched with. We call this problem SMI (SM with Incomplete lists). If a person $p$ 's list includes a person $q$, we say that $q$ is acceptable to $p$. A matching is a disjoint set of pairs $(m, w)$ such that $m$ and $w$ are acceptable to each other. Since we are considering incomplete lists, a matching this time is not necessarily perfect. Hence, we extend the definition of a blocking pair. For a matching $M,(m, w)$ is a blocking pair if the following three conditions are met: (i) $M(m) \neq w$ but $m$ and $w$ are acceptable to each other; (ii) $w \succ_{m} M(m)$ or $m$ is single in $M$; and (iii) $m \succ_{w} M(w)$ or $w$ is single in $M$.

One important property of SMI is that we can partition the set of men (women) into two sets; one is the set of men (women) who have partners in all stable matchings, and the other is the set of men (women) who are single in all stable matchings [14]. (In Sec. 6.2, we will revisit this property in the context of more general Hospitals/Residents problem.) This immediately implies that all stable matchings for a single instance are of the same size. Furthermore, it is easily seen that the Gale-Shapley algorithm with a slight modification can be applied to find a stable matching.

\subsection{Preference Lists with Ties}

The other extension is to allow ties in preference lists, namely, one can include two or more persons with the same preference in a tie. We call this problem SMT (SM with Ties). We extend the notation of preference relation. If women $w_{1}$ and $w_{2}$ are in the same tie of man $m$ 's preference list, we write $w_{1}=_{m} w_{2} \cdot w_{1} \succeq_{m} w_{2}$ means $w_{1} \succ_{m} w_{2}$ or $w_{1}={ }_{m} w_{2}$. Similar notations are used for women's preferences.

In SMT, there are three stability notions: super-stability, strong stability, and weak stability. In the super-stability, a blocking pair is defined as a pair $(m, w)$ such that $M(m) \neq$ $w, w \succeq_{m} M(m)$, and $m \succeq_{w} M(w)$. In the strong stability, $(x, y)$ is a blocking pair if $M(x) \neq y, y \succ_{x} M(x)$, and $x \succeq_{y} M(y)$. Finally, in the weak stability, a blocking pair is defined as $(m, w)$ such that $M(m) \neq w, w \succ_{m} M(m)$, and $m \succ_{w} M(w)$. Note that a super-stable matching is strongly stable, and a strongly stable matching is weakly stable.

It is easy to see that a weakly stable matching always exists and can be found in polynomial time [22]. In contrast, there are instances that have no super-stable nor strongly stable matching. Nevertheless, there is a polynomial time algorithm that decides if a super-stable (strongly stable, resp.) matching exists and finds one if any, whose running time is $O\left(n^{2}\right)$ [22] $\left(O\left(n^{3}\right)\right.$, resp. [42]).

\subsection{Incomplete Preference Lists with Ties}

This extension allows both incompleteness and ties in preference lists. We call this extension SMTI (SM with Ties and Incomplete lists). Definitions of blocking pairs (and hence the stability) can be obtained naturally by combining the two cases of Secs. 3.1 and 3.2. Hence, we have three stability notions again, namely, super, strong, and weak stabilities.

For super and strong stabilities, similar results as SMT hold, namely, there is an algorithm for each case that decides the existence of a stable matching, and finds one if exists. The running time of algorithms are $O(a)$ for superstability [48], and $O(n a)$ for strong stability [42], where $a$ is the total length of all preference lists (which is $2 n^{2}$ if all preference lists are complete). Also, under both super and strong stabilities, all stable matchings for a single instance have the same size, as in the case of SMI.

For the weak stability, a stable matching exists for any instance, and can be found in time $O(a)$, but this time, one instance can have stable matchings of different sizes, and the problem of finding a largest one (which is called MAX $S M T I)$ is NP-hard $[34,50]$. Also, it is known that there is no polynomial time $21 / 19$-approximation algorithm unless $\mathrm{P}=\mathrm{NP}$ [19]. ( $c$-approximation algorithm means that it always finds a stable matching whose size is at least $1 / c$ fraction of the optimal size.)

For approximability, there are a series of improvements $[35,37,38]$, and the current best algorithm achieves the ratio of 1.875 [38]. The same authors [38] improved it to 1.8 (see [43] for example). Also there have been presented approximability and complexity results for instances with 
some restriction, such as the length and/or the position of ties $[18,31,19,30]$.

\section{The Number of Stable Matchings}

As mentioned earlier, any instance of SM has at least one stable matching, but in general, one instance may have a lot. Then, what is the maximum number of stable matchings an instance of size $n$ (i.e. with $n$ men and $n$ women) can have? This problem was first raised by Knuth [44]. Irving and Leather [27] later proved that for any $n$ power of 2 , there is an instance of SM of size $n$ that admits at least $2.28^{n} /(1+\sqrt{3})$ stable matchings. For small $n$, Eilers [11] showed that the maximum number is 10 when $n=4$ by an exhaustive search using computers. However, nontrivial upper bounds are still open. Readers can refer to $[7,61]$ for further information.

\section{Optimal Stable Matchings}

Recall that the Gale-Shapley algorithm finds a stable matching in time $O\left(n^{2}\right)$. This algorithm is basically a sequence of proposals from men to women, and finds a stable matching with an extreme property that every man gets his best possible partner among all stable matchings [13]. In this sense, the matching is called the man-optimal stable matching. Of course, if we exchange the roles of men and women, the resulting stable matching is womanoptimal. Unfortunately, by the nature of stable matchings, the man-optimal stable matching is simultaneously the woman-pessimal stable matching, that is, every woman gets her worst possible partner [46]. Hence, it is natural to try to seek for a matching which is not only stable but also "good" in some criterion.

There are a lot of optimization criteria for the quality of stable matchings, but here we introduce three of them. Let $p_{m}(w)\left(p_{w}(m)\right.$, respectively) denote the position of woman $w$ in man $m$ 's preference list (the position of man $m$ in woman $w$ 's preference list, respectively). For a stable matching $M$, define a regret cost $r(M)$ to be

$$
r(M)=\max _{(m, w) \in M} \max \left\{p_{m}(w), p_{w}(m)\right\} .
$$

Also, define an egalitarian cost $c(M)$ to be

$$
c(M)=\sum_{(m, w) \in M} p_{m}(w)+\sum_{(m, w) \in M} p_{w}(m)
$$

and a sex-equalness cost $d(M)$ to be

$$
d(M)=\sum_{(m, w) \in M} p_{m}(w)-\sum_{(m, w) \in M} p_{w}(m) .
$$

The minimum regret stable marriage problem (the minimum egalitarian stable marriage problem and the sex-equal stable marriage problem, respectively) is to find a stable matching $M$ with minimum $r(M)(c(M)$ and $|d(M)|$, respectively) [16].

Note that the number of stable matchings for one instance grows exponentially in general (see Sec. 4). Nevertheless, for the first two problems, Gusfield [15], and Irving, Leather and Gusfield [28], respectively, proposed polynomial time algorithms by exploiting a lattice structure which is of polynomial size but contains information of all stable matchings. In contrast, the sex-equal stable matching problem is NP-hard [41]. Iwama, et al. gave approximation algorithms for this problem and some variants [39].

If we allow ties in preference lists, the problem of finding an optimal weakly stable matching in any of the above three problems becomes hard, even to approximate: For each problem, there exists a positive constant $\epsilon$ such that there is no polynomial time approximation algorithm with approximation ratio $\epsilon n$ unless $\mathrm{P}=\mathrm{NP}$ [17].

\section{Other Variants}

In this section, we see two major variants of SM, and other related matching problems.

\subsection{Stable Roommates Problem}

The stable roommates problem ( $S R$ for short) is a nonbipartite extension of SM and is defined as follows: We are given an even number $2 n$ of persons, each having a preference list over the other $2 n-1$ people. This problem is to find a stable matching where the stability conditon is defined similarly as the case of SM. In addition to a direct application of assigning people to twin-rooms, SR has applications for pairings of players in chess tournaments [45] and pairwise kidney exchange between incompatible patient-donor pairs [55, 25].

In contrast to the case of SM, there is an instance with no stable matching [13, 16]. Yet, Irving [21] proposed a polynomial time algorithm to decide if an instance admits a stable matching, and finds one if exists. The problem of finding a matching with minimum number of blocking pairs is NP-hard and hard to approximate [2]. If we allow ties in the lists, determining whether an instance admits a weakly stable matching is NP-complete even in complete preference lists [57], while the same problems under the superstability and the strong stability are solved in polynomial time [59, 29].

For SR, a new stability notion, called the exchange stability, was defined and the problem of asking the existence of a stable matching under this stability was proved to be NP-complete [10]. 
The hospitals/residents problem ( $H R$ for short) is a many-to-one extension of SM, where we consider men as residents and women as hospitals. Each hospital declares the quota, that specifies the number of residents the hospital can accept. Usually, in this model, preference lists may be incomplete. As a definition of blocking pairs, we may apply that of SMI by regarding a hospital as single if the number of assigned residents is less than its quota.

We can reduce HR into SM by replacing each hospital with a quota $q$ by its $q$ copies. It is also known that most of the results established for SM hold for HR (see [16]). Here, we would give one remark concerning the property we have seen in Sec. 3.1. This property can be generalized to HR, and is known as the Rural Hospitals Theorem [14, 54]. It says that any stable matching assigns the same number of residents to all hospitals. Furthermore, if a hospital obtains residents fewer than its quota in one stable matching, then the hospital gets the same set of residents in any stable matching.

For some nontrivial results on this problem with ties, see [32] and [33].

\subsection{Other Models}

Finally, in this section, we see some other related problems.

Man-Exchange Stable Marriage For the classical SM, a new stability definition, man-exchange stability was defined. This stability requires, in addition to the original stability, the property that no two men prefer to exchange their partners. Irving have proved that the problem of asking the existence of a man-exchange stable matching is NPcomplete [24].

Many-to-Many Stable Marriage We can consider more general variant than HR, a many-to-many extension of the stable marriage, so that both men and women have quota. One may easily see that the copying technique used in reducing HR to SM cannot be applied any more since if we do so, the resulting stable matching may create multiple copies of the same pair.

Baiou and Balinski [5] showed that some properties for one-to-one and many-to-one case also hold for this case. Bansal et al. [6] gave an efficient algorithm for finding a minimum egalitarian stable matching in this setting. Malhotra [47] studied many-to-many stable marriage with ties, giving an efficient algorithm for finding a strongly stable matching, and also proving that all strongly stable matchings form a distributive lattice.
Student-Project Allocation Problem Student-Project Allocation Problem (SPA) is a variant of HR, in which students are assigned to projects based on his/her preferences over projects. One lecturer may provide two or more projects, and in that case, all projects that are given by the same lecturer have the same preference list. Each project has its own quota, and each lecturer also has his/her quota. We are asked to find a stable matching that satisfies all quota-constraints both for projects and lecturers. Abraham et al. [3] gave two algorithms for solving this problem and also studied its structural properties.

3-Dimensional Stable Matching The 3-dimensional extension of SM was proposed by Knuth [44], in which we are given three sets of agents. There are several freedom in modeling this problem, such as the form of preference lists and the stability definitions. Ng and Hirschberg [53] and Subramanian [60] proposed one model and proved the NP-completeness result. The complexity of another model, called cyclic $3 D$ stable matching, is open but partial results can be found in Boros et al. [8] and Eriksson et al. [12]. Recently, 3-dimensional SR was studied by many groups $[20,36,4]$.

One-Sided Preference Lists There are some matching problems in which only one party (say, men) have preference lists over the other. We introduce here two of them.

A rank-maximal matching (or a greedy matching) is a matching that matches the maximum number of men to their first choice partners, and subject to this condition, the maximum number of men to their second choice partners, and so on. The problem of finding a greedy matching was studied by Irving [23] and Irving et al. [26], who gave polynomial time algorithms.

For two matchings $M_{1}$ and $M_{2}$, if the number of men who prefer $M_{1}$ to $M_{2}$ (in terms of the rank of his partner) is greater than that of men who prefer $M_{2}$ to $M_{1}$, we say that $M_{1}$ is more popular than $M_{2}$. A matching $M$ is popular if there is no matching more popular than $M$. Abraham et al. gave a polynomial time algorithm to decide if a given instance admits a popular matching, and finds a largest one if any [1]. Mestre [51], and Manlove and Sng [49] solved the weighted version and many-one version of this problem, respectively.

\section{Conclusions}

We have explored some results on the stable marriage problem and many of its variants. For further information, readers may refer to the following textbooks $[44,16,56]$. 


\section{References}

[1] D. J. Abraham, R. W. Irving, T. Kavitha, K. Mehlhorn, "Popular matchings," SIAM J. Comput., Vol. 37, No. 4, pp. 1030-1045, 2007.

[2] D. J. Abraham, P. Biró, D. F. Manlove, “ “Almost stable" matchings in the roommates problem," Proc. WAOA 2005, LNCS 3879, pp. 1-14, 2005.

[3] D. J. Abraham, R. W. Irving and D. F. Manlove, "The student-project allocation problem," Proc. ISAAC 2003, LNCS 2906, pp. 474-484, 2003.

[4] E. M. Arkin, A. Efrat, J. S. B. Mitchell, and V. Polishchuk, "Geometric stable roommates," manuscript, 2007.

[5] M. Baiou and M. Balinski, "Many-to-many matching: stable polyandrous polygamy (or polygamous polyandry)," Discrete Applied Mathematics, Vol. 101, pp. 1-12, 2000.

[6] V. Bansal, A. Agrawal and V. S. Malhotra, "Stable marriages with multiple partners: efficient search for an optimal solution," Proc. ICALP 2003, LNCS 2719, pp. 527-542, 2003.

[7] A. T. Benjamin, C. Converse and H. A. Krieger, "How do I marry thee? Let me count the ways," Discrete Applied Mathematics, Vol.59, pp. 285-292, 1995.

[8] E. Boros, V. Gurvich, S. Jastar, and D. Krasner, "Stable matchings in three-sided systems with cyclic preferences," Discrete Mathematics, Vol. 289, pp. 1-10, 2004.

[9] Canadian Resident Matching Service, http://www.carms.ca/

[10] K. Cechlárová, "On the complexity of exchange-stable roommates," Discrete Applied Mathematics, Vol. 116, pp. 279-287, 2002.

[11] D. Eilers, Irvine Compiler Corporation Technical Report, ICC TR1999-2, 1999.

[12] K. Eriksson, J. Sjöstrand, and P. Strimling, "Threedimensional stable matching with cyclic preferences," Mathematical Social Sciences, Vol. 52, pp. 77-87, 2006.

[13] D. Gale and L. S. Shapley, "College admissions and the stability of marriage," Amer. Math. Monthly, Vol.69, pp. 9-15, 1962.

[14] D. Gale and M. Sotomayor, "Some remarks on the stable matching problem," Discrete Applied Mathematics, Vol.11, pp. 223-232, 1985.

[15] D. Gusfield, "Three fast algorithms for four problems in stable marriage," SIAM J. Comput., Vol. 16, Issue 1, pp. 111-128, 1987.
[16] D. Gusfield and R. W. Irving, "The Stable Marriage Problem: Structure and Algorithms," MIT Press, Boston, MA, 1989.

[17] M. M. Halldórsson, R. W. Irving, K. Iwama, D. F. Manlove, S. Miyazaki, Y. Morita, and S. Scott, "Approximability Results for Stable Marriage Problems with Ties", Theoretical Computer Science, Vol. 306, pp. 431-447, 2003.

[18] M. M. Halldórsson, K. Iwama, S. Miyazaki and H. Yanagisawa, "Randomized approximation of the stable marriage problem," Theoretical Computer Science, Vol. 325, No. 3, pp. 439-465, 2004.

[19] M. M. Halldórsson, K. Iwama, S. Miyazaki, and H. Yanagisawa, "Improved approximation results of the stable marriage problem," ACM Transactions on Algorithms, Vol. 3, Issue 3, Article No. 30, 2007.

[20] C. C. Haung, "Two's company, three's a crowd: stable family and threesome roommates problems," Proc. ESA 2007, LNCS 4698, pp. 558-569, 2007.

[21] R. W. Irving, "An efficient algorithm for the "stable roommates" problem," J. Algorithms, Vol. 6, No. 4, pp. 577-595, 1985.

[22] R. W. Irving, "Stable marriage and indifference," Discrete Applied Mathematics, Vol.48, pp. 261-272, 1994.

[23] R. W. Irving, "Greedy matchings," University of Glasgow, Computing Science Department Research Report, TR-2003-136, 2003.

[24] R. W. Irving, "Man-exchange stable marriage," University of Glasgow, Computing Science Department Research Report, TR-2004-177, 2004.

[25] R. W. Irving, "The cycle roommates problem: a hard case of kidney exchange," Information Processing Letters, Vol. 103, Issue 1, pp. 1-4, 2007.

[26] R. W. Irving, T. Kavitha, K. Mehlhorn, D. Michail, and K. Paluch, "Rank-maximal matchings," Proc. SODA 2004, pp. 68-75, 2004.

[27] R. W. Irving and P. Leather, "The complexity of counting stable marriages," SIAM J. Comput., Vol.15, pp. 655-667, 1986.

[28] R. W. Irving, P. Leather and D. Gusfield, "An efficient algorithm for the "optimal" stable marriage," Journal of the ACM, Vol. 34, pp. 532-543, 1987.

[29] R. W. Irving and D. F. Manlove, "The stable roommates problem with ties," J. Algorithms, Vol. 43, No. 1, pp. 85-105, 2002.

[30] R. W. Irving and D. F. Manlove, "An 8/5approximation algorithm for a hard variant of stable marriage," Proc. COCOON 2007, LNCS 4598, pp. 548-558, 2007. 
[31] R. W. Irving, D. F. Manlove and G. O'Malley, "Stable marriage with ties and bounded length preference lists," Proc. the 2nd Algorithms and Complexity in Durham workshop, Texts in Algorithmics, College Publications, 2006.

[32] R. W. Irving, D. F. Manlove, S. Scott, "The hospitals/residents problem with ties," Proc. SWAT 2000, LNCS 1851, pp. 259-271, 2000.

[33] R. W. Irving, D. F. Manlove, S. Scott, "Strong stability in the hospitals/residents problem," Proc. STACS 2003, LNCS 2607, pp. 439-450, 2003.

[34] K. Iwama, D. F. Manlove, S. Miyazaki, and Y. Morita, "Stable marriage with incomplete lists and ties," Proc. ICALP 99, LNCS 1644, pp. 443-452, 1999.

[35] K. Iwama, S. Miyazaki, and K. Okamoto, "A (2 $c \log N / N)$-approximation algorithm for the stable marriage problem," Proc. SWAT 2004, LNCS 3111, pp. 349-361, 2004.

[36] K. Iwama, S. Miyazaki, and K. Okamoto, "Stable roommates problem with triple rooms," Proc. 10th KOREA-JAPAN Joint Workshop on Algorithms and Computation (WAAC 2007), pp. 105-112, 2007.

[37] K. Iwama, S. Miyazaki, N. Yamauchi, "A $\left(2-c \frac{1}{\sqrt{N}}\right)$ approximation algorithm for the stable marriage problem," Proc. ISAAC 2005, LNCS 3827, pp. 902-914, 2005 .

[38] K. Iwama, S. Miyazaki, N. Yamauchi, “A 1.875approximation algorithm for the stable marriage problem," Proc. SODA 2007, pp. 288-297, 2007.

[39] K. Iwama, S. Miyazaki, and H. Yanagisawa, "Approximation algorithms for the sex-equal stable marriage problem," Proc. WADS 2007, LNCS 4619, pp. 201213, 2007.

[40] Japan Residency Matching Program, http://www.jrmp.jp/

[41] A. Kato, "Complexity of the sex-equal stable marriage problem," Japan Journal of Industrial and Applied Mathematics (JJIAM), Vol. 10, pp. 1-19, 1993.

[42] T. Kavitha, K. Mehlhorn, D. Michail, and K. Paluch, "Strongly stable matchings in time $O(\mathrm{~nm})$ and extension to the H/R problem," Proc. STACS 2004, pp. 222233, 2004.

[43] S. Khuller, "Problems column," ACM Transactions on Algorithms, Vol. 3, Issue 3, Article No. 35, 2007.

[44] D. E. Knuth, "Mariages Stables," Les Presses de l'Université Montréal, 1976. (Translated and corrected edition, "Stable Marriage and Its Relation to Other Combinatorial Problems," CRM Proceedings and Lecture Notes, Vol. 10, American mathematical Society, 1997.
[45] E. Kujansuu, T. Lindberg, and E. Mäkinen, "The stable roommates problem and chess tournament pairings," Divulgaciones Matemáticas, Vol. 7, No. 1, pp. 19-28, 1999.

[46] D. McVitie and L. B. Wilson, "The stable marriage problem," Communications of the ACM, Vol. 14, pp. 486-490, 1971.

[47] V. S. Malhotra, "On the stability of multiple partner stable marriages with ties," Proc. ESA 2004, LNCS 3221, pp. 508-519, 2004.

[48] D. F. Manlove, "Stable marriage with ties and unacceptable partners," University of Glasgow, Computing Science Department Research Report, TR-199929, 1999.

[49] D. F. Manlove and C. T. S. Sng, "Popular matchings in the capacitated house allocation problem," Proc. ESA 2006, LNCS 4168, pp. 492-503, 2006.

[50] D. F. Manlove, R. W. Irving, K. Iwama, S. Miyazaki, and Y. Morita, "Hard variants of stable marriage," Theoretical Computer Science, Vol. 276, Issue 1-2, pp. 261-279, 2002.

[51] J. Mestre, "Weighted popular matchings," Proc. ICALP 2006, pp. 715-726, 2006.

[52] National Resident Matching Program, http://www.nrmp.org/

[53] C. Ng and D. S. Hirschberg, "Three-dimensional stable matching problems," SIAM Journal on Discrete Mathematics, Vol. 4, pp. 245-252, 1991.

[54] A. E. Roth, "The evolution of the labor market for medical interns and residents: a case study in game theory", J. Political Economy, Vol. 92, No. 6, pp. 9911016, 1984.

[55] A. E. Roth, T. Sonmez, and M. U. Unver, "Pairwise kidney exchange," Journal of Economic Theory, Elsevier, Vol. 125, No. 2, pp. 151-188, 2005.

[56] A. E. Roth and M. Sotomayor, "Two-sided Matching: A Study in Game-theoretic Modeling and Analysis," Cambridge University Press, Cambridge, 1991.

[57] E. Ronn, "NP-complete stable matching problems," J. Algorithms, Vol. 11, pp. 285-304, 1990.

[58] Scottish PRHO Allocations, http://www.dcs.gla.ac.uk/rwi/SPA.html

[59] S. Scott, "A study of stable marriage problems with ties," Ph.D. Thesis, University of Glasgow, 2005.

[60] A. Subramanian, "A new approach to stable matching problems," SIAM J. Comput., Vol. 23, No. 4, pp. 671701, 1994.

[61] E. G. Thurber, "Concerning the maximum number of stable matchings in the stable marriage problem," Discrete Mathematics, Vol.248, pp. 195-219, 2002. 